\title{
Numerical Study of Flow Field and Aerodynamic Performance of Straight Bladed VAWT at Variable Tip Speed Ratios
}

\author{
Mei Yi* and Qu Jianjun \\ School of Mechatronics Engineering, Harbin Institute of Technology, Harbin, Heilongjiang, 15001, China
}

\begin{abstract}
This paper studies the relationship between unsteady flow features and instantaneous torque and power performance of straight bladed vertical axis wind turbine at variable tip speed ratios. The rotor unsteady flow field simulation was carried out by using computational fluid dynamics method. The flow physics and the principle of changing flow field acting on torque performance and power performance have been analyzed where the rotating rotor was the major concern. The results show that the flow feature alters from periodical blade dynamic stall vortexes generation, development and shedding at low tip speed ratio to cyclical formation, evolution and diffusion of blade wake flow with the rising tip speed ratio. Both vortex shedding around the blade and interaction of blade wakes degrade the rotor aerodynamic performance. It is suggested that, to absorb maximum wind energy, delay the blade vortex shedding and reduce the range of blade wake, evolution and diffusion should be included in the rotor aerodynamic design.
\end{abstract}

Keywords: Computational fluid dynamics, Flow field, Power coefficient, Vertical axis wind turbine, Model geometry, Key parameters, Computational domain.

\section{INTRODUCTION}

Modern wind turbines are classified into horizontal axis wind turbines (HAWTs) and vertical axis wind turbines (VAWTs) based on their axis of rotation. HAWTs are better suited for applying in large wind farms. However, VAWTs, especially straight bladed VAWTs have begun to play a major role in wind power in recent years, because there is a growing interest in harvesting wind energy within the

environment built in urban sites [1-3]. There can be advantages for using straight bladed VAWT machines in urban areas. Primarily, straight bladed VAWTs have no need for yawing system which simplifies the structure, are easy to manufacture because blades can be of constant profile along the span, and have relatively small noise emission level due to lower operating blade tip speeds. All these merits are important in building the environment and in this connection straight bladed VAWTs are good candidate for urban wind energy utilization.

It is well known that flow field of VAWTs is characterized by unsteady flow features. The unsteady blade flow physics has been investigated by Martinat [4] and Wang [5] by simulating the flow around an airfoil with rapid changes in the angle of attack. Research performed by Qin [6] shows the dynamic stall characteristics of VAWT blade. PIV studies conducted by Ferreira [7] revealed complex mechanisms of dynamic stall phenomenon of VAWT blade. Yang [8] analyzed the relationship between velocity distribution and blade aerodynamic forces by numerical approach. Gao [9] studied the influence of flow features on blade dynamic performance. Though previous researches on

*Address correspondence to this author at the School of Mechatronics, Harbin Institute of Technology, Harbin, Heilongjiang, 150001, China; Tel: 0086-13654543250; E-mail: darrenymei@gmail.com
VAWT flow field have achieved various degrees of success, limitations still can be found. Previous studies focused on flow features around single blade and the influence of unsteady flow phenomenon on blade lift performance and drag performance. As the rotating of wind turbine is driven by multiple blades, the aerodynamic performance of wind turbine is directly reflected by power and torque performance of wind rotor and blades, which are concerned with the application of VAWT. Moreover, flow features cannot be the same at different blade tip speed ratios. Therefore, the connection between flow structures, and power and torque performance of VAWT needs to be investigated at variable tip speed ratios.

In this paper, a comprehensive study conducted on relationships between unsteady flow features and instantaneous torque and power performance of VAWT is investigated by CFD technique, which can display detailed flow physics and calculate aerodynamic characteristics, provided theoretical guidance to the engineering application of straight bladed VAWTs.

\section{NUMERICAL METHODOLOGY}

\subsection{Model Geometry and Key Parameters}

The straight bladed VAWT used in the present study is a typical machine applied in urban sites. The design is shown in Fig. (1). This model is a three straight bladed VAWT model. The rotor has a diameter of $d=2 \mathrm{~m}$ with a central shaft diameter of $d_{c}=0.1 \mathrm{~m}$. The blade chord length and rotor height are $c=0.35 \mathrm{~m}$ and $H=2.5 \mathrm{~m}$, respectively. The choice of blade profile is NACA0015, which is the widely used airfoil in many commercialized straight bladed VAWTs. Assuming the wind speed in urban regions is usually between $6 \mathrm{~m} / \mathrm{s}$ to $10 \mathrm{~m} / \mathrm{s}$ [10], the wind velocity value in this study was maintained at $8 \mathrm{~m} / \mathrm{s}$. 


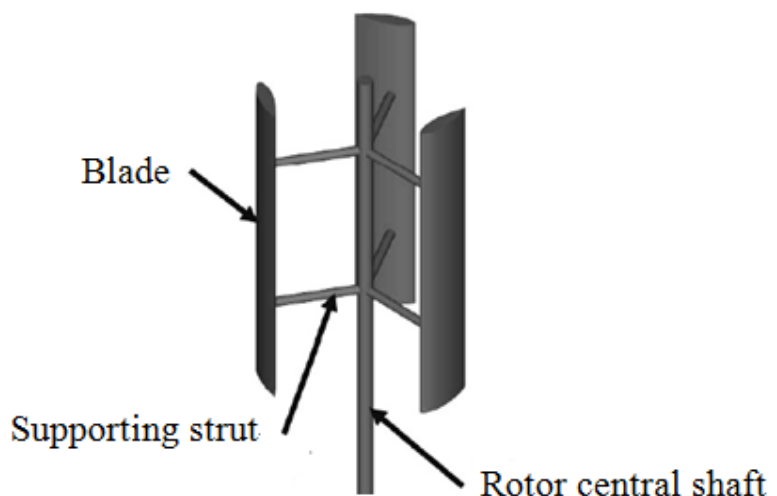

Fig. (1). Diagrammatic sketch of a three-bladed rotor.

The tip speed ratio $(\lambda)$ is a ratio between the tip blade speed and the free stream wind velocity, and is defined as:

$\lambda=\frac{\omega R}{V_{\infty}}$

where $\omega$ is the rotor rotating angular velocity, $R$ is the rotor radius, and $V_{\infty}$ is the free stream velocity.

Power coefficient $\left(C_{P}\right)$ written as Eq. (2) is an expression of turbine harness wind energy, and torque coefficient $\left(C_{T}\right)$ described by Eq. (3) reveals the torque performance of the rotor.

$C_{P}=\frac{2 T_{a v e} \omega}{\rho S V_{\infty}^{3}}$

where $T_{\text {ave }}$ is the averaged torque of wind rotor during each revolution, $\rho$ is the density of the air, $S$ is the swept area of wind turbine.

$C_{T}=\frac{2 T_{a v e}}{\rho S R V_{\infty}^{2}}$

\subsection{Control Equations and Turbulence Model}

The code uses the finite volume method to solve the governing equations for fluids. For VAWT, the incompressible unsteady Reynolds Averaged Navier-Stokes (URANS) equations, formulating the principles of conservation of mass, momentum and energy in the form of partial differential equations, were solved for the rotor flow field. Control equations can be written in general form as:

$\frac{\partial(\rho \Phi)}{\partial t}+\nabla(\rho V \Phi)=\nabla(\Gamma V \Phi)+S$

The shear stress transport (SST) k- $\omega$ model, developed by Menter [11], has been successfully applied for previous numerical simulations of $\mathrm{H}$ type VAWT by other researchers [12-14]. Therefore, the SST k- $\omega$ model was employed for all the simulations presented in this study. The governing equations are given by

$\frac{\partial(\rho k)}{\partial t}+\frac{\partial\left(\rho U_{j} k\right)}{\partial x_{j}}=\tilde{P}_{k}-E_{k}+\frac{\partial\left(\Gamma_{k} \frac{\partial k}{\partial x_{j}}\right)}{\partial x_{j}}$

$$
\begin{aligned}
\frac{\partial(\rho \omega)}{\partial t}+\frac{\partial\left(\rho U_{j} \omega\right)}{\partial x_{j}}=P_{\omega}-E_{\omega}+D_{\omega}+\frac{\partial\left(\Gamma_{\omega} \frac{\partial \omega}{\partial x_{j}}\right)}{\partial x_{j}} & \frac{\partial(\rho \gamma)}{\partial t}+\frac{\partial\left(\rho U_{j} \gamma\right)}{\partial x_{j}}=P_{\gamma 1}-E_{\gamma 1}+P_{\gamma 2}-E_{\gamma 2} \\
& +\frac{\partial\left[\frac{\partial \gamma}{\partial x_{j}}\left(\mu+\frac{\mu_{t}}{\sigma_{\gamma}}\right)\right]}{\partial x_{j}} \\
\frac{\partial\left(\rho \tilde{\operatorname{Re}} e_{\theta t}\right)}{\partial t}+\frac{\partial\left(\rho U_{j} \tilde{R} e_{\theta t}\right)}{\partial x_{j}}= & \frac{\partial\left[\frac{\partial \tilde{\operatorname{R}} e_{\theta t}}{\partial x_{j}} \sigma_{\theta t}\left(\mu+\mu_{t}\right)\right]}{\partial x_{j}}
\end{aligned}
$$

where $\tilde{P}_{k}, P_{\omega}, P_{\gamma 1}, E_{\gamma 1}$ are the production terms of the kinetic energy, specific dissipation and transition sources. $D_{\omega}$ is the cross diffusion term. $E_{k}, E_{\omega}, P_{\gamma 2}, \mathrm{E}_{\gamma_{2}}$ are the destruction terms of the kinetic energy, specific dissipation and transition sources. $P_{\theta t}$ is the source term.

\subsection{Computational Domain}

A full two-dimensional turbine model of the straight bladed VAWT is created on the basis of the geometry mentioned previously. As illustrated in Fig. (2), the domain is created for use in the computations by assembling a circular rotating region in a rectangular stationary region where the rectangular stationary region is $15 d$ in length and $10 d$ in width, and the diameter of circular rotating region is $2 d$. The upstream side is set as an inlet boundary condition whereas the downstream side is set as a pressure outlet boundary condition with two wall boundaries to both sides of the rectangular. The boundary where rectangular region connects to circular rotating region is set as an interface boundary condition to employ the sliding mesh technique.

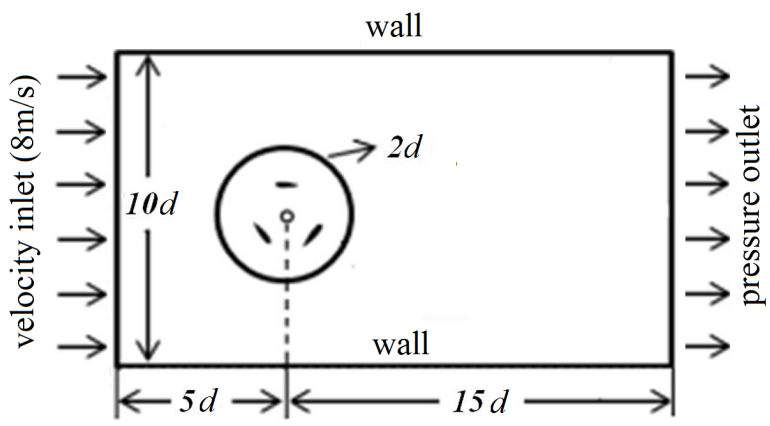

Fig. (2). Computational domain.

As shown by Fig. (3), O type mesh was adopted around each blade. A mesh independence test was performed. Several different structured grids of increasing density and quality, composed of various mesh size ranging from 100,000 up to 700,000 cells are investigated. Result shows that more than 600,000 cells lead to a relative variation of the output quantity below $1 \%$. Based on the grid independence test, the height of the first cell used is $1.07 \times 10^{-4}$ $\mathrm{m}$, the growth rate of inflation is implemented as 1.07 to give an average of the $y$ plus values from the flow solutions of 5 to 10 , and the total number of cells in the domain is 600348 . 


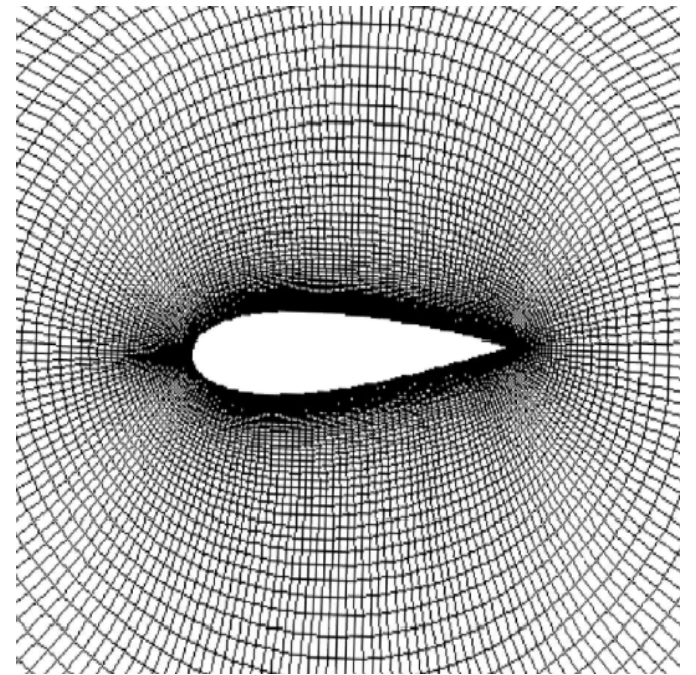

Fig. (3). Mesh around the blade.

The coupled pressure based solver was selected with a second order implicit transient formulation for improved accuracy. The PISO algorithm was applied for the solution of the pressure-velocity coupled equations. All solution variables were solved via QUICK scheme. All the residuals are set to $10^{-5}$ for all the variables.

\section{RESULTS AND ANALYSIS}

Fig. (4a) shows the calculated power performance of rotor. As $\lambda$ ranges from 0.5 to 3.5 , the power coefficient increases steadily before the data reaches the highest point of 0.312 at $\lambda=2.5$, then drops to 0.252 at $\lambda=3.5$. Instantaneous torque coefficient $\left(C_{\text {Tbinst }}\right)$ curves are depicted in Fig. (4b) and periodic changes can be observed. The widest fluctuation comes from $C_{\text {Tbinst }}$ curve at $\lambda=0.5$. More smooth profiles of curve and data values are observed at $\lambda=2.5$ and $\lambda=3.5$. The difference is related to the change of unsteady flow field. In order to illustrate the influence of the changing unsteady flow field on power and torque performance, instantaneous torque coefficient and power coefficient in conjunction with flow field at three tip speed ratios $(\lambda=0.5$, $\lambda=2.5$ and $\lambda=3.5$ ) will be analyzed in the following part.

\subsection{Blade Flow Field at Variable Tip Speed Ratios}

Fig. (5) shows the blade vorticity contours during one cycle at $\lambda=0.5$. Vortexes generating and shedding can be observed around the blade. This is because the flow velocity is relatively small at lower tip speed ratio, leading to flow separation and dynamic stall. At $\theta=60^{\circ}$, vortexes which are about to shed from the blade surface, can be seen at leading edge and trailing edge. At $\theta=90^{\circ}$, a large scale vortex is shedding from the blade and secondary vortexes are generated at leading and trailing edge. From $\theta=120^{\circ} \sim 180^{\circ}$, the scale of newly generated leading edge vortex increases gradually and sheds from the blade whereas the trailing edge vortex spreads to the leading edge and separates and sheds from the blade. In the downwind region $\left(\theta=180^{\circ} \sim 360^{\circ}\right)$, because of the lower flow velocity, vortexes generate and shed more frequently. The flow does not attach to the blade surface until $\theta=360^{\circ}$. (a)

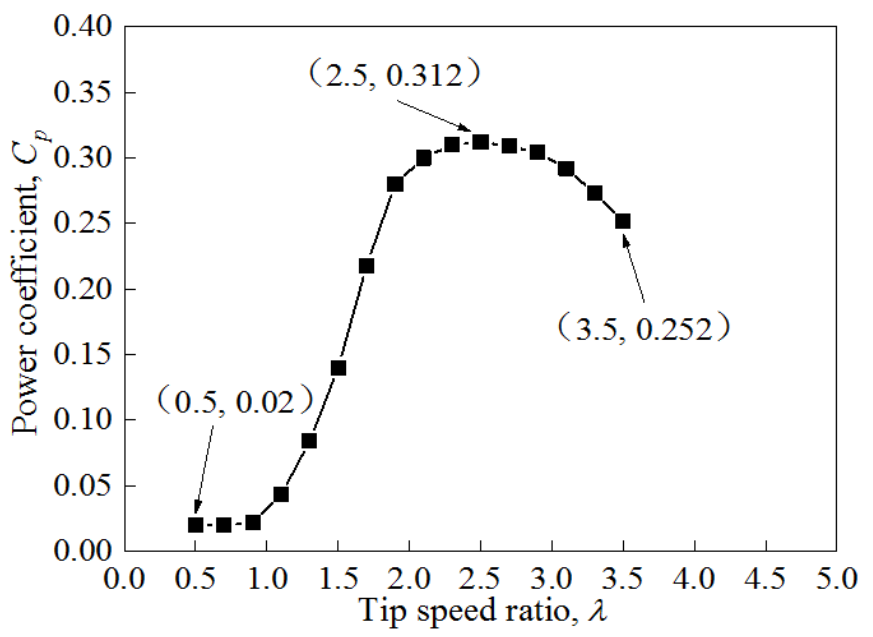

(b)

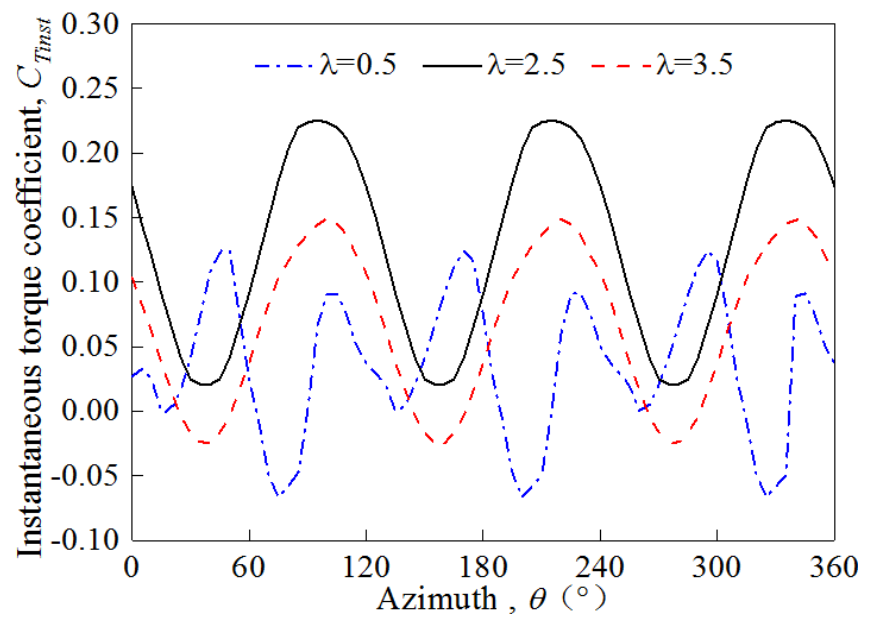

Fig. (4). (a) calculated power performance; (b) instantaneous rotor torque performance at three tip speed ratios.

The blade instantaneous torque coefficient $C_{\text {Tbinst }}$ and power coefficient $C_{\text {Pbinst }}$ rose at $\lambda=0.5$ are shown by Fig. (6). The same trend can be observed for the two curves. $C_{\text {Tbinst }}$ and $C_{\text {Pbinst }}$ are maintain positively in the range of $\theta=30^{\circ} \sim 60^{\circ}$ and $\theta=90^{\circ} \sim 120^{\circ}$. During the two scopes, vortexes are generated at both leading edge and trailing edge of the blade, resulting in a low pressure region existing at the suction surface, which can delay the stall and enhance the aerodynamic performance of blade. This is the reason why values of $C_{\text {Tbinst }}$ and $C_{\text {Pbinst }}$ attain peak point. When dynamic stall vortex sheds from the blade surface, there is a dramatic decrease in the boundary layer momentum, weakening the aerodynamic performance. $C_{\text {Tbinst }}$ and $C_{\text {Pbinst }}$ are negative in the range of $\theta=60^{\circ} \sim 90^{\circ}$ and $\theta=120^{\circ} \sim 150^{\circ}$.

Fig. (7) shows the blade vorticity contours at different azimuths during one cycle at $\lambda=2.5$. The flow features are different from the one illustrated in Fig. (5), that is no significant dynamic stall phenomenon can be observed. This is due to the flow momentum increases with the rising tip speed ratio, overcoming adverse pressure gradient and making boundary layer well attached to the blade surface. It can be seen in the figure that only small scale vortex is 

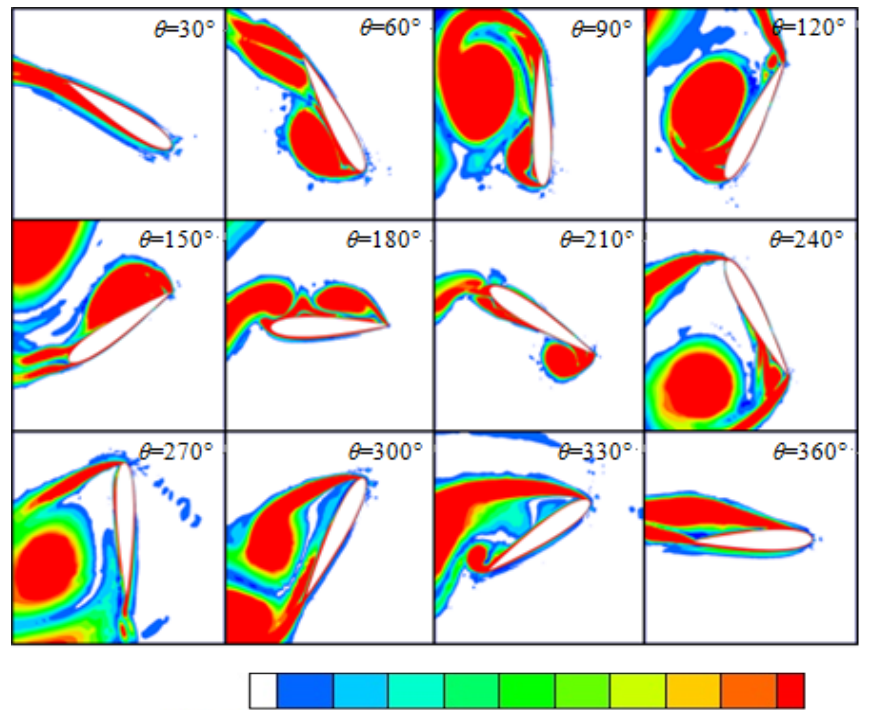

Vorticity (1/s) 50100150200250300350400450500

Fig. (5). Vortex contours of single airfoil on different positions at $\lambda=0.5$.

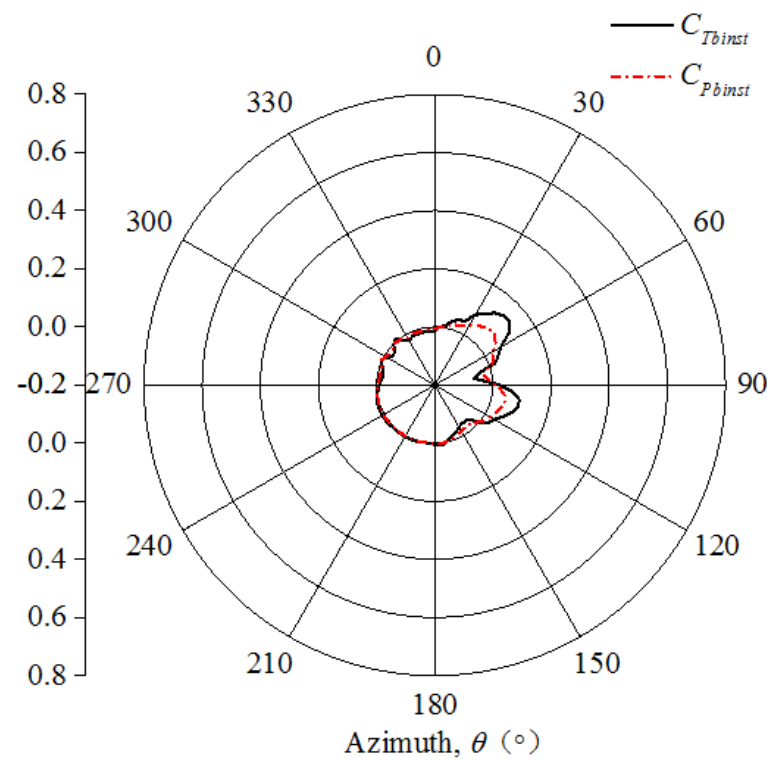

Fig. (6). Blade instantaneous torque coefficient and power coefficient at $\lambda=0.5$.

generated with trailing edge separation in the range of $\theta=150^{\circ} \sim 180^{\circ}$. The flow is also stable in the downwind region.

$C_{\text {Tbinst }}$ and $C_{\text {Pbinst }}$ rose at $\lambda=2.5$ as shown in Fig. (8). In comparison with Fig. (6), the change of coefficients is steady and the curves are smoother. As there is no prominent dynamic stall phenomenon, the values of $C_{\text {Tbinst }}$ and $C_{\text {Pbinst }}$ at $\lambda=2.5$ are higher than those at $\lambda=0.5$. It should be noted that the level of CTbinst and CPbinst in the downwind region is much lower than the one in the upwind region, indicating that the blade in upwind region absorbs the majority wind energy when the air is flowing toward the wind rotor and no energy can be harnessed in the downwind region, which provides the explanation for the phenomenon that the windenergy utilization efficiency level keeps lower in downwind region though no prominent dynamic stall and flow separation are detected.

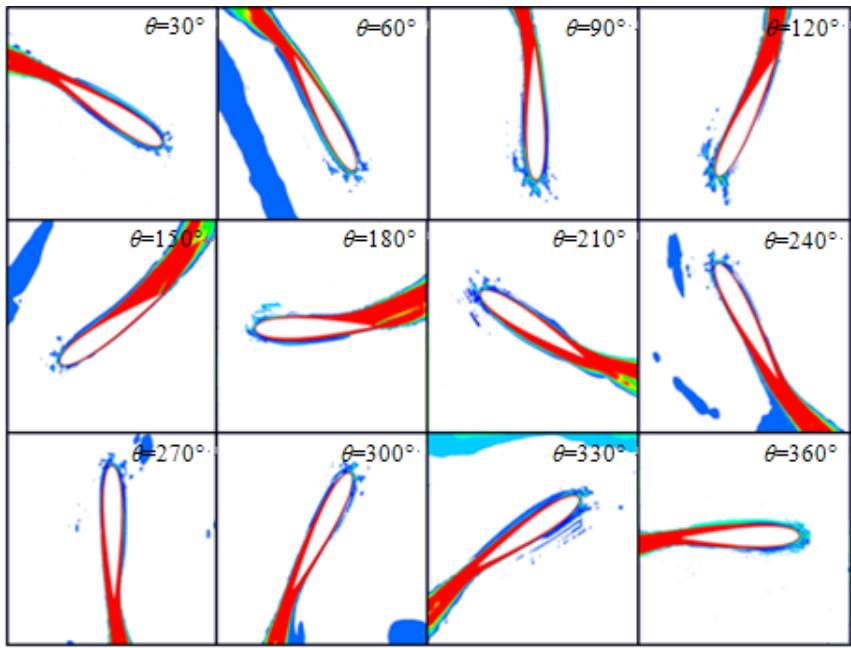

Vorticity (1/s) 50100150200250300350400450500

Fig. (7). Vortex contours of single airfoil on different positions at $\lambda=2.5$.

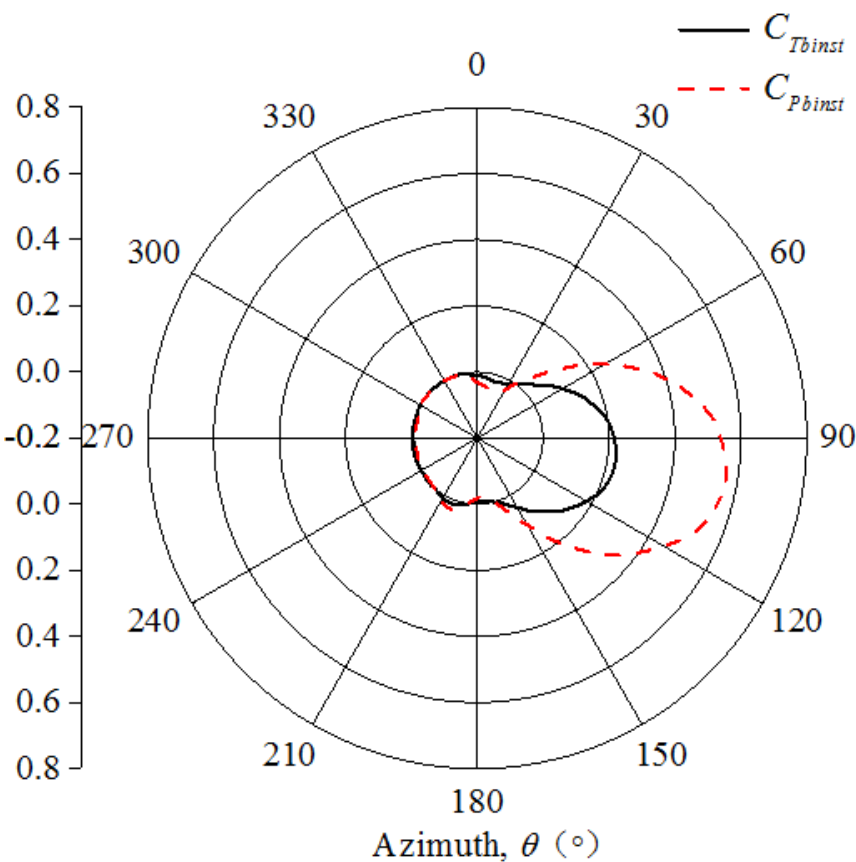

Fig. (8). Blade instantaneous torque coefficient and power coefficient at $\lambda=2.5$.

The instantaneous vorticity contours plotted for various angels at $\lambda=3.5$ are illustrated in Fig. (9). It is observed that vorticity contours exhibit significant difference between $\lambda=0.5$ and $\lambda=2.5$. Dynamic vortex can be barely found during the blade rotation in a circle, which is similar to the case of $\lambda=2.5$. However, blade vortex is seriously disturbed by other blades. Blade evades the wake flow of the former blade during the rotation of blade positioned at $\theta=30^{\circ}, \theta=180^{\circ}$ and $\theta=360^{\circ}$, separately, while blade positioned at $\theta=60^{\circ}$ avoids the wake disturbance by the former blade. As vortex contours of $\theta=150^{\circ}$ and $\theta=330^{\circ}$ are indicated, blades enter the wake flow of the former blade for the second and third time. The variation of blade performance is dominated not only by the formation and shedding of vortex, but also by the wake flow. 
Fig. (10) demonstrates $C_{\text {Tinst }}$ and $C_{\text {Pinst }}$ rose for the blade at various rotation angles. As it can be seen from the figure, it is noted that both values of $C_{\text {Tbinst }}$ and $C_{\text {Pinst }}$ are minus and show the same rising trend for the blade rotating from $\theta=0^{\circ} \sim 30^{\circ}$, which is attributed to the wake flow effect. While for the blade located at azimuth angles ranging from $\theta=30^{\circ} \sim 150^{\circ}$, performance curves correlated with $C_{\text {Tbinst }}$ and $C_{P i n s t}$ are similar in shape or changing trend with $\lambda=2.5$ case, i.e. both values of $C_{\text {Tbinst }}$ and $C_{\text {Pinst }}$ keep rising until reaching the top, and then drop down. Aerodynamic performance of blade rotating from $\theta=150^{\circ}$ is substantially weakened from the dual effects brought by the disturbance of other blades as well as the significant

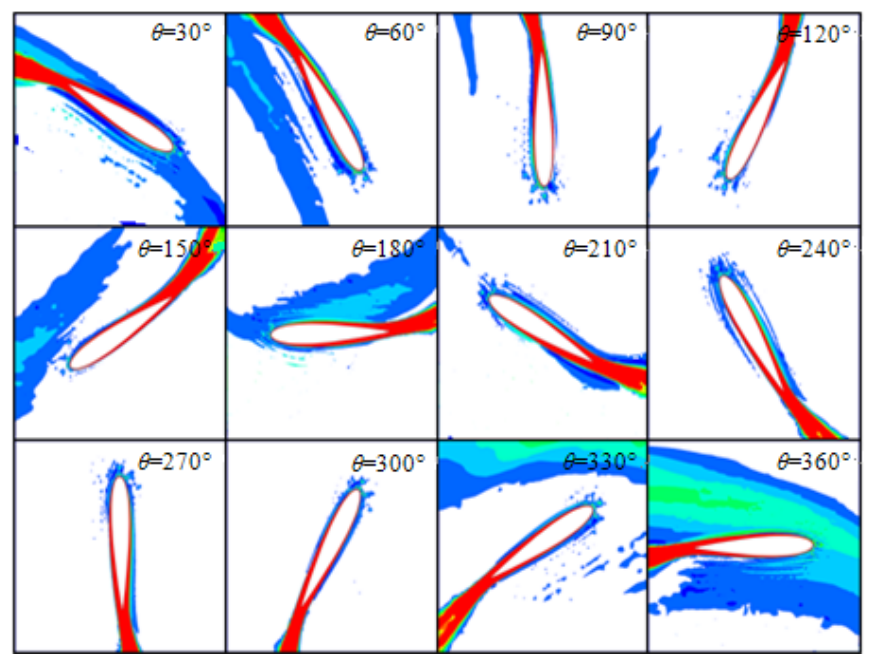

Vorticity (1/s) 50100150200250300350400450500

Fig. (9). Vortex contours of single airfoil on different positions at $\lambda=3.5$.

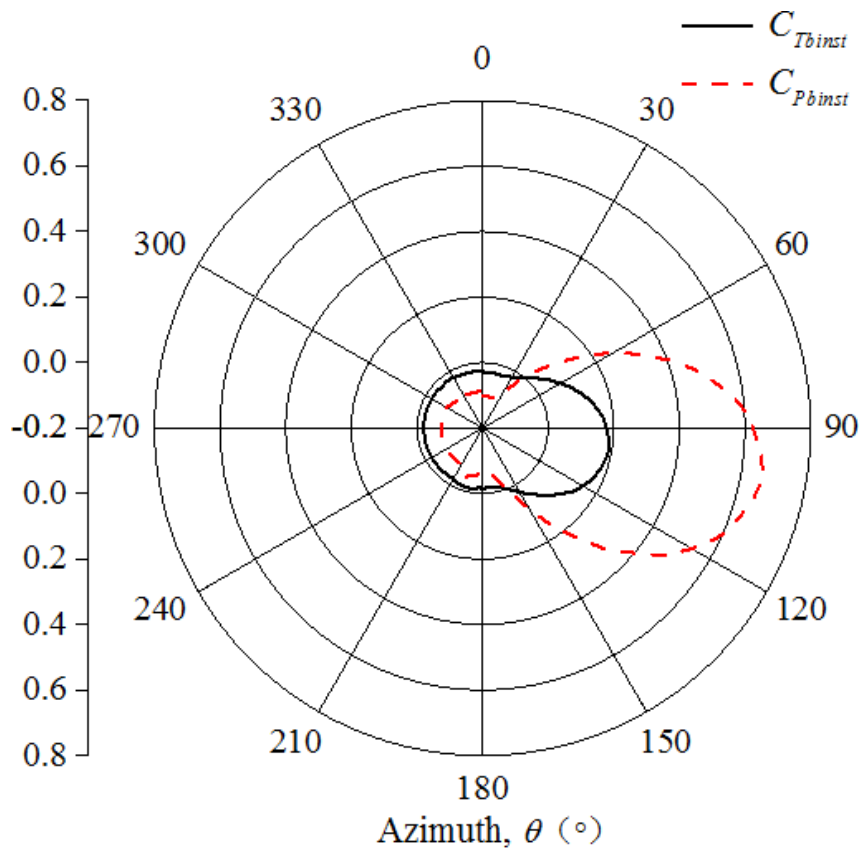

Fig. (10). Blade instantaneous torque coefficient and power coefficient at $\lambda=3.5$. reduction of air-motion energy absorbed by blade invading the downwind region. CTbinst aerodynamic performance of blade rotating from $\theta=150^{\circ}$ is substantially weakened from, $C_{\text {Tbinst }}$ and $C_{\text {Pinst }}$ are all minus for blade rotating from $\theta=30^{\circ} \sim 150^{\circ}$. Hence, it can be deduced that the activation torque is transformed by the absorbed wind energy by the blade in the upwind region.

\subsection{Rotor Flow Field at Variable Tip Speed Ratios}

Fig. (11) illustrates the velocity contour of rotor rotating at every $60^{\circ}$ of a turning circle with $\lambda=0.5$. As can be seen, the obvious dynamic stall is observed on the surface of blade, which can be ascribed to the fact that the fluid velocity is relatively small due to the low tip-speed ratio, and then after the separation of blade surface from boundary layer, vortex can be formed by the downstream movement in the flow fields. When the blade locates between $\theta=0^{\circ}$ and $\theta=120^{\circ}$, there is large separation vortex on the blade surface of Blade 1, leading to the appearance of vortex at the leading edge and tale part of the blade as well. And then vortex scale increases with the rotation till it being dropped off, while on the blade surface of Blades 2 and 3, the generation, spreading and shedding processes of vortex occur repeatedly. As for the stages separately starting from $\theta=0^{\circ} \sim 30^{\circ}$ and ranging from $\theta=60^{\circ}$ until $\theta=90^{\circ}$, vortex shedding appears around the Blades 1 and 2 in the upwind region, seriously deteriorating the aerodynamic performance of the wind turbine. While vortex is forming on the blade surface of Blade 1 in the upwind region, vortex is already generated again after the former vortex shedding. When the rotation is located at position with azimuth angles of $\theta=30^{\circ}$ and $\theta=60^{\circ}$, it indicates that low pressure region is emerging from the blade surface, which is beneficial for enhancing the aerodynamic performance. This can also be observed for the rotation position locating at $\theta=0^{\circ} \sim \theta=30^{\circ}$.

Fig. (12) shows instantaneous rotor torque coefficient $C_{\text {Tinst }}$ and instantaneous power coefficient $C_{\text {Pinst }}$ rose for the turbine following any one blade, which reveals that both the values have a 3-period-variation, with both the values separately reaching peaks and troughs twice for each circle. Take the first circle for consideration, when the blade moves to the position locating ranging from $\theta=30^{\circ} \sim 60^{\circ}$ and $\theta=60^{\circ} \sim 90^{\circ}$, vortex is proceeding to the process of generation and development, and simultaneously low pressure region is formed on the surface of the blade, resulting in the vortexformation delay, which consequently improves the aerodynamic performance, and thus, values of $C_{\text {Tbinst }}$ and $C_{P b i n s t}$ peaks at the azimuth angle interval. Comprehensively, vortex generates, spreads, sheds and repeats the circle constantly attributed to the relatively low tip-speed ratio. The wind energy can only be employed sub-efficiently, so $C_{P i n s t}$ keeps lower than 0.1 .

Fig. (13) shows the vorticity contour at various positions for the wind turbine at $\lambda=2.5$. As can be seen from the picture, it is noted that the three blades of the wind turbine are all characterized by the wake flow evidently. The reason can be explained as follows: once dynamic air moves by the turbine, energy of motion can be absorbed by boundary layer of the blade, which definitely could decelerate the motion velocity of air. This is verified by the velocity contour 


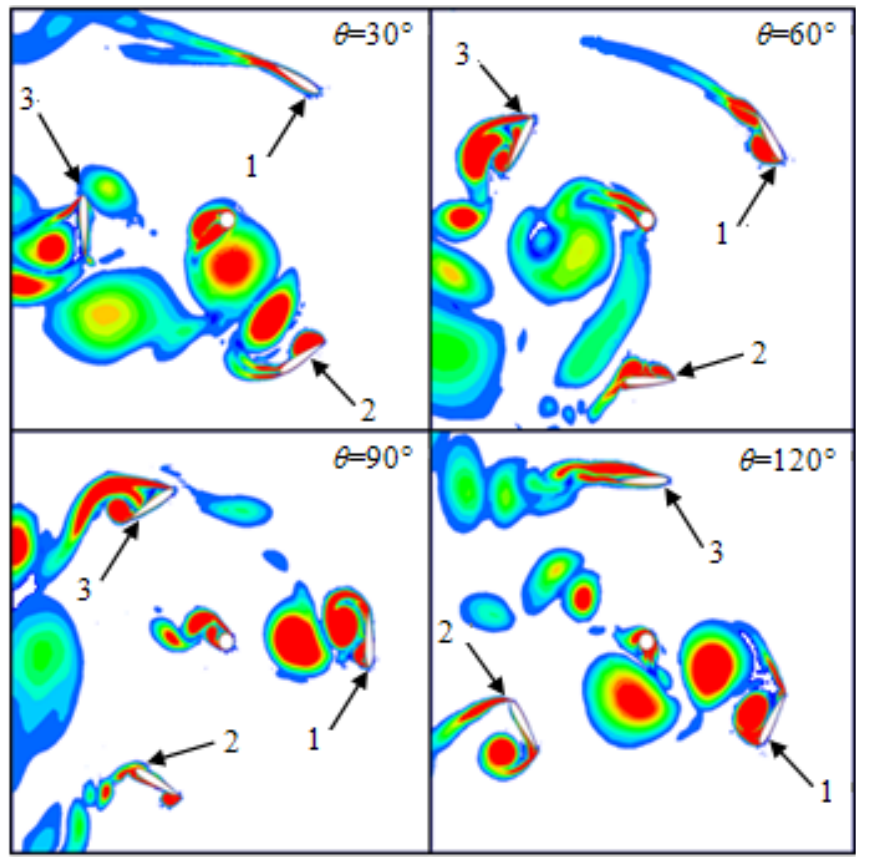

Vorticity (1/s) 50100150200250300350400450500

Fig. (11). Vortex contours of rotor $\lambda=0.5$.

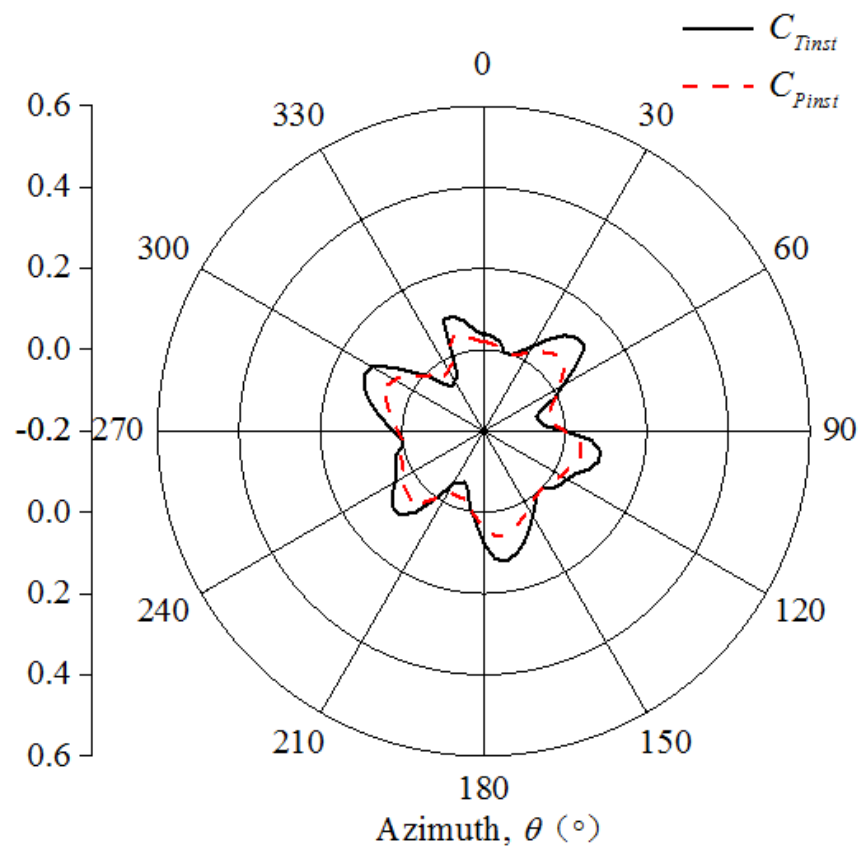

Fig. (12). Rotor instantaneous torque coefficient and power coefficient at $\lambda=0.5$.

illustrated in Fig. (14), showing that wake-flow scale of the Blade 1 gradually increases in the upwind region, while wake-flow scale weakens after Blade 3 rotates into the downwind region. This is because the velocity gradient is conducive to energy exchange between wake flow and air flow around, i.e., mixing wake flow and airflow contributes to elongation of track in the blade's wake. Air-mixture area expands leading to the broadening of the wake flow, and therefore velocity gap is gradually reduced in the wake.

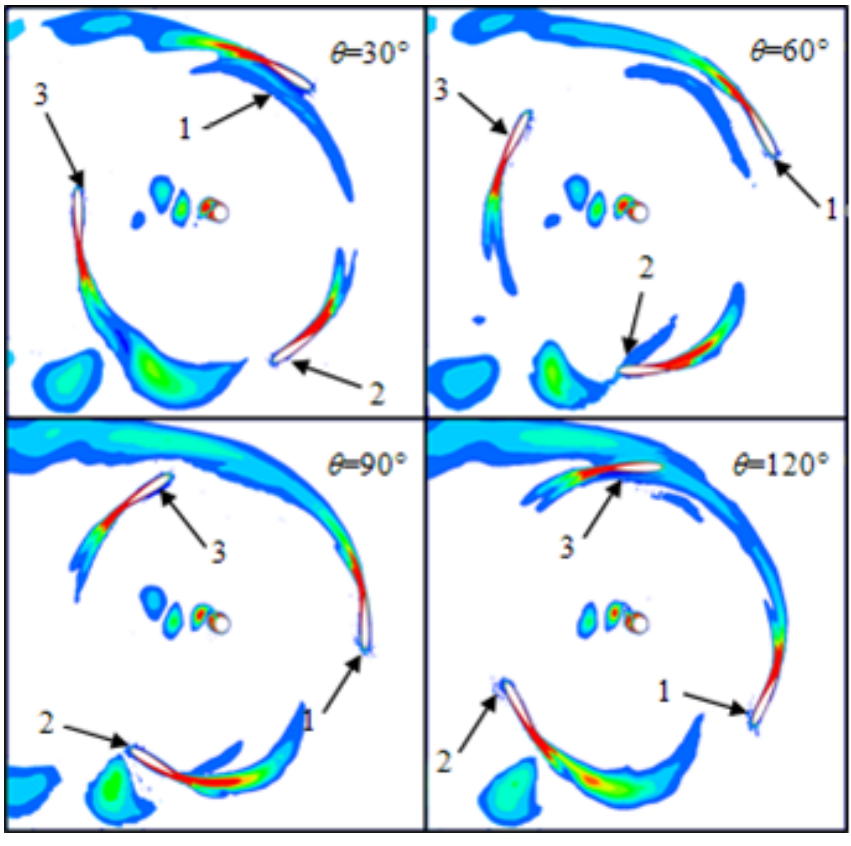

Vorticity (1/s) 50100150200250300350400450500

Fig. (13). Vortex contours of rotor $\lambda=2.5$.
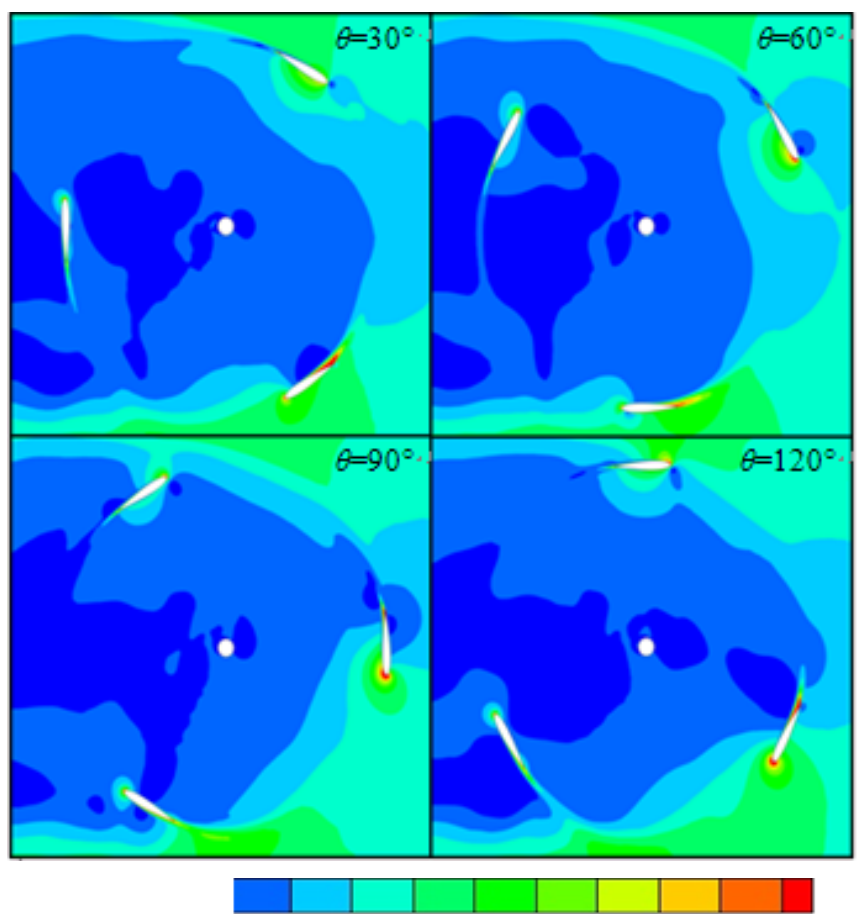

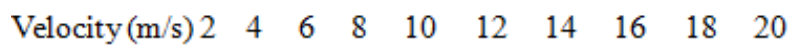

Fig. (14). Velocity contours of rotor $\lambda=2.5$.

Fig. (15) shows the calculated $C_{\text {Tinst }}$ and $C_{\text {Pinst }}$ that rose at $\lambda=2.5$. It is found that $C_{\text {Tinst }}$ and $C_{\text {Pinst }}$ are much higher and the curves are also much smoother compared to that of $\lambda=2.5$, suggesting aerodynamic performance becomes more stable given the increment of the tip-speed ratio. However, airmotion energy is depleted partially by the wake flow of blade, and hence, wake flow becomes longer and broader, leading consequently to the turbine in the downwind region 
rotating into the upwind region, which has weakened the aerodynamic performance of the upwind turbine. The wake flow of the rotating blade joins in the generating and diffusive circles, and it is the reason why $C_{\text {Tinst }}$ and $C_{\text {Pinst }}$ retain at the topmost and the lowest points each once from the changing curves.

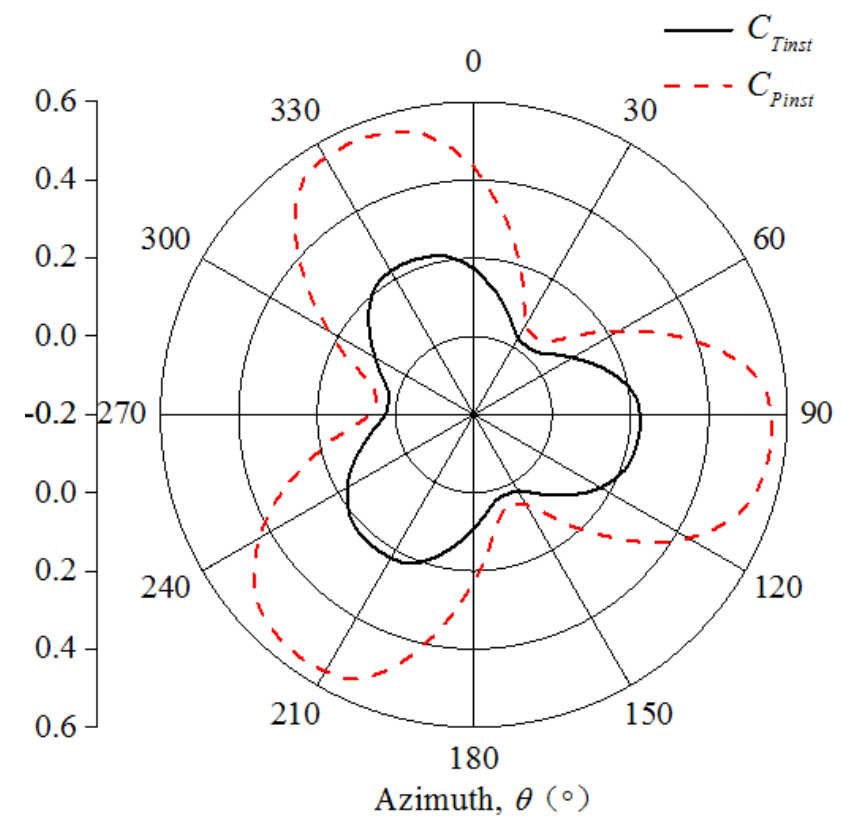

Fig. (15). Rotor instantaneous torque coefficient and power coefficient at $\lambda=2.5$.

Vorticity contour and velocity contours of rotor at $\lambda=3.5$ are shown in Figs. $(\mathbf{1 6}, \mathbf{1 7})$. It is clearly seen that in comparison with the vortex image of $\lambda=2.5$ case, with

the
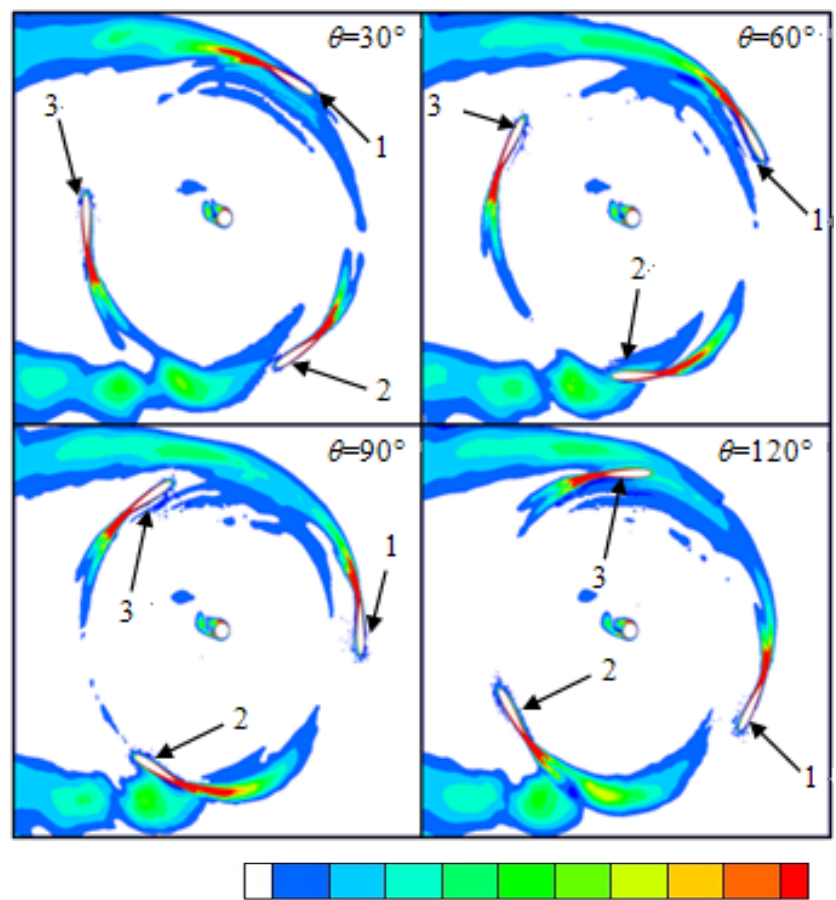

Vorticity (1/s) 50100150200250300350400450500

Fig. (16). Vortex contours of rotor $\lambda=3.5$.

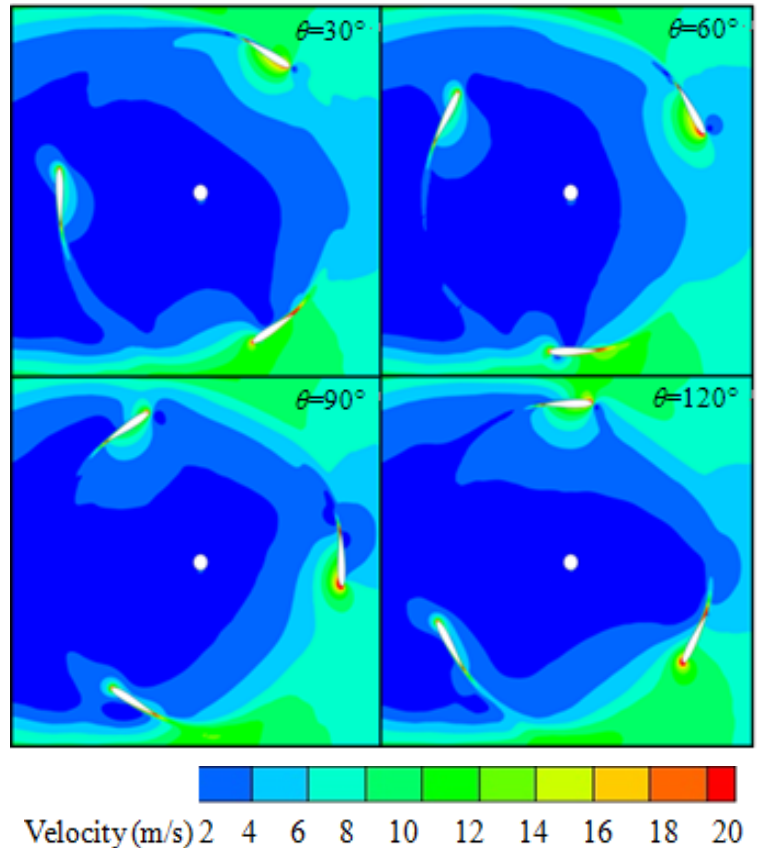

Fig. (17). Velocity contours of rotor $\lambda=3.5$.

further increase in tip-speed ratio, the wake flow effect is more intense. Indeed, at azimuth angle ranging from $\theta=30^{\circ} \sim 120^{\circ}$, the wake flow is much longer than that at $\lambda=2.5$, The diffusive track becomes much broader for the wake flowing toward the air around, which gives rise to downwind turbine being effected at relatively large angle interval. As can be seen from Fig. (17), compared to the $\lambda=2.5$ case, the further increment of tip-speed ratio makes velocity gradient from the wake center to the region both around the upwind blade and around the downwind blade more obvious. It suggests that the attenuation of wake flow is slowed down, and thus velocity gap between the wake flow and flow around cannot be instantaneously diminished, which can be extended toward downwind region. The trend details are given in Fig. (17), The wake still exists after Blade 2 and Blade 3 enter the downwind region of turbine, particularly, Blade 2, during its rotation from $\theta=30^{\circ} \sim 90^{\circ}$, is continuously affected by Blade 3 . The strong wake effect causes the disturbance more intense in the rotor flow field, which has a negative influence on the torque and power.

Fig. (18) presents the predicated $C_{\text {Tinst }}$ and $C_{\text {Pinst }}$ rose for the rotor with $\lambda=3.5$ at various rotation angles. As compared with the $\lambda=2.5$ counterpart, it is seen that apparently the performance behaviors featured by closed geometric are observed in both the cases. As tip speed ratio is elevated to 3.5 , performance curves shrink toward the center, that is to say, the minimum values of $C_{\text {Tinst }}$ and $C_{\text {Pinst }}$ are relatively lower for a periodical rotatio, and moreover the maximum values of $C_{\text {Tinst }}$ and $C_{\text {Pinst }}$ are smaller than that of $\lambda=2.5$ case, due possibly to the strong wake-flow effect that has weakened the aerodynamic performance of wind turbine. The decreasing wind velocity caused by blade consuming air-motion energy should be responsible for the wake flow by referring to the above-mentioned analysis. The intense wake flow of the turbine is indicative of less energy obtained by blade from the air movement, Thus the smaller the torque transformed to be applied to activate wind-turbine motion, and the smaller the $C_{\text {Pinst }}$ is accordingly for $\lambda=2.5$ case. In 
addition, the wake flow gradually grows longer and diffuses outward followed by the generation and spread, which could disturb and delay the rotation of other blades, so both values of $C_{\text {Tinst }}$ and $C_{\text {Pinst }}$ decrease sharply to the minus level.

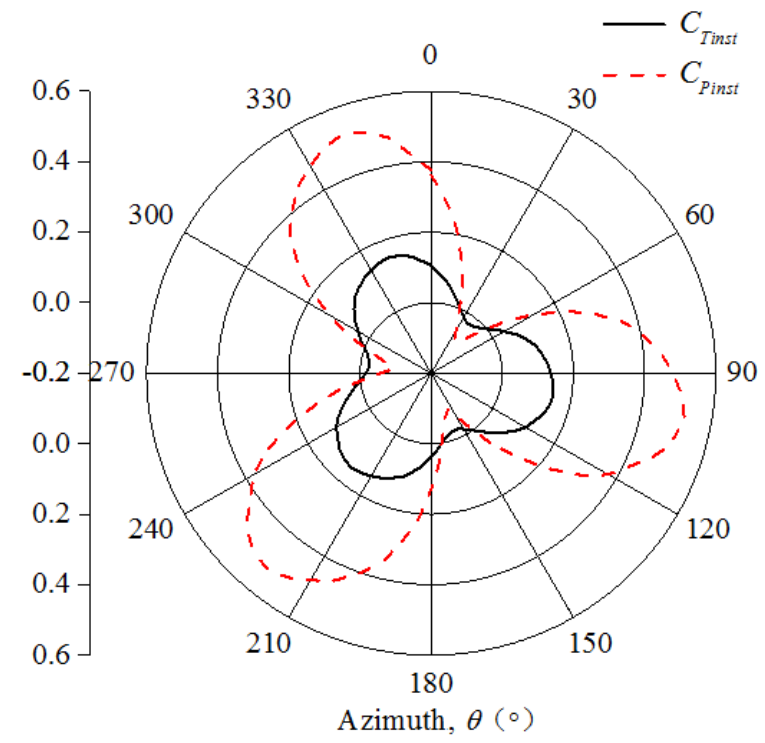

Fig. (18). Rotor instantaneous torque coefficient and power coefficient at $\lambda=3.5$.

\section{CONCLUSION}

The unsteady flow feature is influenced by changing tip speed ratio, which results in the change of rotor power performance and torque performance. The rotor driven forces mainly come from the wind energy that rotor harness in the upwind region. At lower tip speed ratio, the flow field features with cyclic frequent dynamic stall and flow separation, which lead to relatively lower level of torque and power coefficients. At higher tip speed ratio, the periodic blade wake generating and spreading are the major features that can be observed in the flow field. The interaction of blade wakes has negative influence on torque and power performance. Therefore, in order to absorb the maximum wind energy, delaying the blade vortex shedding and reducing the range of blade wake evolution and diffusion should be included in the straight bladed VAWT rotor aerodynamic design.

\section{CONFLICT OF INTEREST}

The authors confirm that this article content has no conflict of interest.

\section{ACKNOWLEDGEMENTS}

Declared none.

\section{REFERENCES}

[1] D. R. Drew, J. F. Barlow, and T. T. Cockeril, "Estimating the potential yield of small wind turbines in urban areas: A case study for Greater London UK", J. Wind Eng. Ind. Aerodyn., vol. 115, pp. 104-111, 2013.

[2] F. T. Silva, A. C. Santos, and M. C. Gil, "Urban wind energy exploitation systems: Behavior under multidirectional flow conditions opportunities and challenges", Renew. Sust. Energ. Rev., vol. 24, pp. 364-378, 2013.

[3] J. D. Booker, P. H. Mellor, R. Wrobel, and D. Drury, "A compact high efficiency contra-rotating generator suitable for wind turbines in the urban environment", Renew. Energ., vol. 35, pp. 2027-2033, 2010 .

[4] G. Martinat, M. Braza, Y. Hoarau, and G. Harran, "Turbulence modeling of the flow past a pitching NACA0012 airfoil at $10^{5}$ and $10^{6}$ Reynolds numbers", J. Fluid Struct., no. 24, pp. 1294-1303, 2008.

[5] S. Y. Wang, D. B. Ingham, L. Ma, M. Pourkashanian, and Z. Tao, "Numerical investigations on dynamic stall of low Reynolds number flow around oscillating airfoils", Comput. Fluids, vol. 39, no. 9, pp. 1529-1541, 2010.

[6] N. Qin, R. Howell, N. Durrani, K. Hamada, and T. Smith, "Unsteady flow simulation and dynamic stall behavior of vertical axis wind turbine blades", Wind Eng., vol. 35, no. 4, pp. 511-527, 2011.

[7] C. S. Ferreira, G. V. Bussel, G. A. Kuiket, F. C. J. Simao, and V. B. S Fulvio, "On the use of velocity data for load estimation of a VAWT in dynamic stall", J. Sol. Energ. Eng., vol. 133, pp. 0110061-0110068, 2011.

[8] Z. Q. Yang, and W. Z. Cui, "Numerical simulation for 2D unsteady flow around the vertical axis wind turbine", Mod. Manuf. Eng., no. 3, pp. 56-59, 2009. (in Chinese).

[9] W. Gao, C. Li, Z. Ye, and J. B. Nie, "Dynamic characteristics and aerodynamic performance of vertical axis wind turbine", J. Drain. Irrig. Mach. Eng., vol. 32, no. 2, pp. 146-150, 2014. (in Chinese).

[10] L. H. Zhao, M. Liu, and T. Lv, "Study on the aerodynamic performance of blade airfoil of vertical axis wind turbine at low Reynolds number", Inf. Technol. J., vol. 12, no. 14, pp. 3042-3045, 2013.

[11] F. R. Menter, "Two equation eddy viscosity turbulence models for engineering applications", AIAA J., vol. 32, no.8, pp. 1598-1605, 1994.

[12] K. M. Almohammadi, D. B. Ingham, L. Ma, and M. Pourkashanian, "2D CFD analysis of the effect of trailing edge shape on the performance of a straight blade vertical axis wind turbine", IEEE T. Sustain. Energ., vol. 6, no.1, pp. 228-234, 2015.

[13] M. R. Castelli, A. Englaro, and E. Benini, "The darrieus wind turbine: proposal for a new performance prediction model based on CFD”, Energy, vol. 36, pp. 4919-4934, 2011.

[14] O. Gueeri, A. Sakout, and K. Bouhadef, "Simulations of the fluid flow around a rotating vertical axis wind turbine", Wind Eng., vol. 31, no. 3, pp. 149-163, 2007. 